\title{
Competing risk nomogram to predict cancer-specific survival in esophageal cancer during the intensity-modulated radiation therapy era: A single institute analysis
}

\author{
SHENGGUANG ZHAO, WEIXIANG QI and JIAYI CHEN \\ Department of Radiation Oncology, Rui Jin Hospital Affiliated Medicine School \\ of Shanghai Jiao Tong University, Shanghai 200025, P.R. China \\ Received Seotember 13, 2019; Accepted January 21, 2020
}

DOI: $10.3892 / \mathrm{ol} .2020 .11448$

\begin{abstract}
The present study aimed to investigate the probability of cancer-associated mortality of patients with esophageal cancer undergoing intensity-modulated radiation therapy (IMRT), and to establish a competing risk nomogram to predict the esophageal cancer-specific survival (EC-SS) of these patients. A total of 213 patients with EC who underwent IMRT between January 2014 and May 2017 were selected to establish nomograms according to Fine and Gray's competing risk analysis. Predictive accuracy and discriminative ability of the model were determined using the concordance index (C-index), calibration curves and the area under receiver operating characteristic curves. Decision tree analysis was also constructed for patient grouping. With a median follow-up of 19 months (range, 3-50), the 2-year EC-specific mortality (EC-SM) and the non-esophageal cancer specific mortality (NEC-SM) of the cohort were 35.4 and $3.51 \%$, respectively. Furthermore, an elevated 2-year EC-SM was observed in patients with tumor length $\geq 4.5 \mathrm{~cm}$ compared with patients with tumor length $<4.5 \mathrm{~cm}(45.8 \%$ vs. $21.4 \%$; $\mathrm{P}<0.001)$, patients with non-squamous cell carcinoma compared with patients with squamous cell carcinoma (49.9 vs. 33.7\%; $\mathrm{P}=0.025)$ and patients with $\mathrm{N} 3$ stage (43.2\%; $\mathrm{P}=0.005)$. The 2-year NEC-SM of patients with tumor length $\geq 4.5 \mathrm{~cm}$ was $6 \%$ vs. $0 \%$ in patients with tumor length $<4.5 \mathrm{~cm}$ $(\mathrm{P}=0.016)$. Three independent risk factors for survival, including tumor length, histological type and $\mathrm{N}$ stage, were integrated to build competing nomograms for the EC-SS
\end{abstract}

Correspondence to: Dr Jiayi Chen or Dr Weixiang Qi, Department of Radiation Oncology, Rui Jin Hospital Affiliated Medicine School of Shanghai Jiao Tong University, 197 Second Ruijin Road, Shanghai 200025, P.R. China

E-mail: chenjiayi0188@aliyun.com

E-mail: qiweixiang1113@163.com

Key words: esophageal cancer, competing risk nomogram, intensity-modulated radiation therapy, tumor length, prognosis model $(\mathrm{C}$-index $=0.72 ; 95 \%$ confidence interval, 0.66-0.77). In addition, the nomograms displayed better discrimination power than the 7 th edition of the Tumor-Node-Metastasis staging system for predicting EC-SS (area under the curve $=0.707$ vs. 0.634). Furthermore, the results from the classification tree analysis demonstrated that $\mathrm{N}$ stage was the initial node and that primary tumor length was a determinant for EC-SM in these patients. In conclusion, NEC-SM represented a competing event for patients with EC with a tumor length $\geq 4.5 \mathrm{~cm}$. The competing risk nomograms may therefore be considered as convenient individualized predictive tools for cancer-specific survival in patients with EC undergoing IMRT treatment.

\section{Introduction}

Esophageal cancer (EC) was the 7th most diagnosed malignant tumor $(572,000$ new cases) and the 6th leading cause of cancer-associated mortality (509,000 mortality cases) worldwide in 2018 (1). The incidence of EC varies considerably among geographical regions and sexes. EC mostly occurs in men ( $70 \%$ of all cases), and there is a $2-3$-fold difference in the incidence and mortality rates between regions worldwide (2). In the latest report from 2017, EC ranked the 6th most common type of cancer diagnosed and the 4th leading cause of cancer-associated mortality in China $(3,4)$. At present, esophagectomy is considered as the standard treatment for patients with resectable EC (5). However, 50\% of patients present with locally advanced disease at the time of diagnosis (6). Definitive radiation therapy with or without concurrent chemotherapy has been recommended as the optimal treatment for patients who are medically inoperable or have locally advanced disease (7). Intensity-modulated radiotherapy (IMRT) has been widely used for the treatment of EC, because it can provide excellent dose coverage and conformity to the target volume while minimizing excessive dose to healthy tissues, compared with 3D conformal radiotherapy (8). In addition, the number of elderly patients with $\mathrm{EC}$ has grown in the last decades due to the increasing life expectancy and population aging. Elderly patients with EC are more vulnerable to experience competing events, including non-cancer-associated mortality (9). It is therefore crucial to 
clearly evaluate the risk factors for cancer-specific survival in patients with EC receiving IMRT.

Cancer patients are frequently exposed to $>2$ events different from the one of interest and are identified as competing risk events (10). In the presence of competing risks, conventional survival analysis may be inappropriate as this type of analysis considers competing events as censored events, which might overestimate the incidence of cancer-associated mortality $(11,12)$. Recently, competing risk analysis has been widely used in cancer research as it takes into account the informative nature of censoring and may more effectively discriminate the effects of risk factors on specific events $(13,14)$. A nomogram is a statistical model that generates individualized risk prediction by combining risk factors (15). Competing risk nomograms have been recently developed for various types of cancer, including renal cell carcinoma (16), nasopharyngeal carcinoma (17), breast cancer (18), thyroid cancer (19) and lung cancer (14). However, to the best of our knowledge, a competing risk nomogram for EC following IMRT is still lacking. The present study therefore performed a competing risk analysis based on a cohort of patients with EC undergoing IMRT. Subsequently, competing nomograms were established to provide clinicians with a quantitative tool that can be used to evaluate the probability of EC-specific mortality (EC-SM) and non-esophageal cancer specific mortality (NEC-SM) in order to better stratify the risk and make the best clinical decision.

\section{Materials and methods}

Study population. The medical records of patients with EC who underwent IMRT at the Shanghai Rui Jin Hospital Affiliated to Shanghai Jiao Tong University School of Medicine between January 2014 and May 2017 were recovered. The inclusion criteria were as follows: i) Diagnosis of EC was confirmed independently by two pathologists through hematoxylin and eosin staining in a retrospective review; ii) complete medical records containing the clinicopathological characteristics of the patients were available; iii) no history of previous or concurrent malignancy; and iv) follow-up period of $>3$ months following IMRT. A total of 213 patients with EC undergoing IMRT in the Department of Radiation Oncology of Rui Jin Hospital Affiliated Medicine School of Shanghai Jiao Tong University were subsequently identified and included in the present study. All patients received standard IMRT of 50.4 Gy (28 fractions, 1.8 Gy per fraction, in 5.6 weeks) as the primary treatment. When possible, salvage treatments, including surgery and re-irradiation, were provided for patients with relapsed or persistent disease. The present study was approved by the Institutional Review Board at Shanghai Rui Jin Hospital Affiliated to Shanghai Jiao Tong University School of Medicine. As the present study was retrospective in nature, the requirement for written informed consent from the patients was waived.

Clinical variables and definitions. Clinicopathological characteristics of patients included sex, age at diagnosis, date of diagnosis, tumor site, performance status, tumor length,
Table I. Clinicopathological characteristics of the 213 patients with esophageal cancer.

\begin{tabular}{|c|c|}
\hline Clinical feature & Patient number $(n=213)(\%)$ \\
\hline \multicolumn{2}{|l|}{ Sex, n (\%) } \\
\hline Male & $183(85.9)$ \\
\hline Female & $30(14.1)$ \\
\hline Age (years), median (range) & $63(41-87)$ \\
\hline \multicolumn{2}{|l|}{ Histological type, n (\%) } \\
\hline $\mathrm{SCC}$ & 191(89.7) \\
\hline Non-SCC & $22(10.3)$ \\
\hline \multicolumn{2}{|l|}{ Performance status, n (\%) } \\
\hline 0 & $82(38.5)$ \\
\hline $1-2$ & $131(61.5)$ \\
\hline \multicolumn{2}{|l|}{ Tumor location, n (\%) } \\
\hline Cervical & $7(3.3)$ \\
\hline Thoracic & $131(61.5)$ \\
\hline Abdominal & $75(35.2)$ \\
\hline $\begin{array}{l}\text { Tumor length }(\mathrm{cm}), \text { median } \\
\text { (range) }\end{array}$ & $5(1-16)$ \\
\hline \multicolumn{2}{|l|}{ Grade, n (\%) } \\
\hline Well & $17(8.0)$ \\
\hline Moderate & $107(50.2)$ \\
\hline Poor/undifferentiated & $89(41.8)$ \\
\hline \multicolumn{2}{|l|}{ T stage, n (\%) } \\
\hline $\mathrm{T} 1$ & $8(3.8)$ \\
\hline $\mathrm{T} 2$ & $25(11.7)$ \\
\hline $\mathrm{T} 3$ & $135(63.4)$ \\
\hline $\mathrm{T} 4$ & $45(21.1)$ \\
\hline \multicolumn{2}{|l|}{$\mathrm{N}$ stage, n (\%) } \\
\hline NO & $39(18.3)$ \\
\hline $\mathrm{N} 1$ & $63(29.6)$ \\
\hline $\mathrm{N} 2$ & $60(28.2)$ \\
\hline N3 & $51(23.9)$ \\
\hline \multicolumn{2}{|l|}{ M stage, n (\%) } \\
\hline Yes & $25(11.7)$ \\
\hline No & $188(88.3)$ \\
\hline \multicolumn{2}{|l|}{ AJCC stage, n (\%) } \\
\hline I & $8(3.8)$ \\
\hline II & $41(19.2)$ \\
\hline III & $86(40.4)$ \\
\hline IV & $78(36.6)$ \\
\hline \multicolumn{2}{|l|}{ Concurrent chemoradiotherapy } \\
\hline Yes & $172(80.8)$ \\
\hline No & $41(19.2)$ \\
\hline \multicolumn{2}{|l|}{ Radical surgery } \\
\hline Yes & $104(51.2)$ \\
\hline No & $109(48.8)$ \\
\hline
\end{tabular}

AJCC, American Joint Committee on Cancer; SCC, squamous cell carcinoma; T, tumor; $\mathrm{N}$, node; $\mathrm{M}$, metastasis.

grade, pathological diagnosis, tumor stage at the time of diagnosis, concurrent chemoradiotherapy, primary tumor 
A

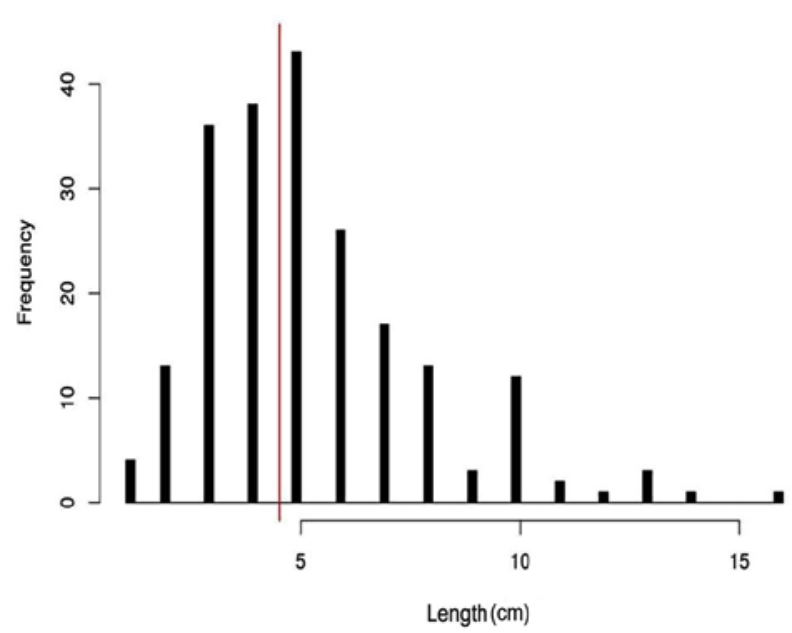

C

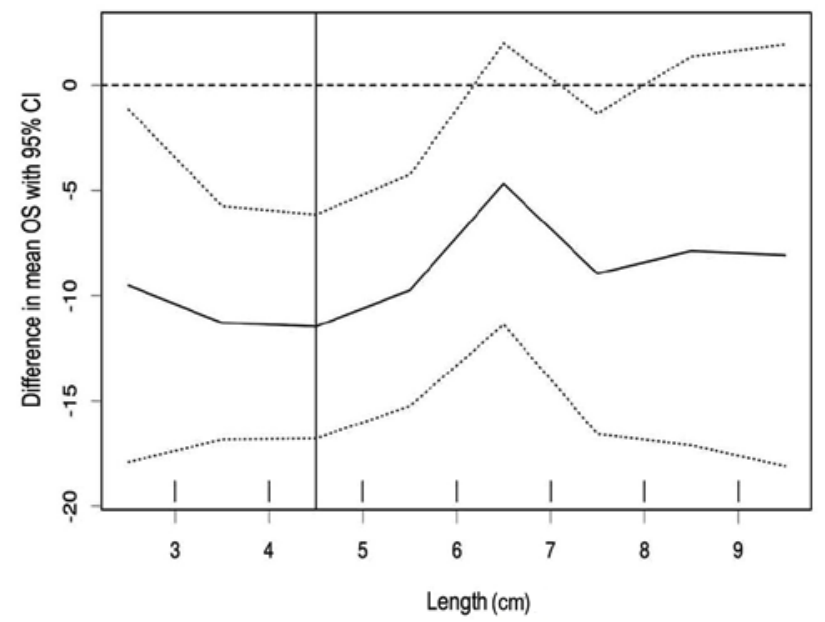

B

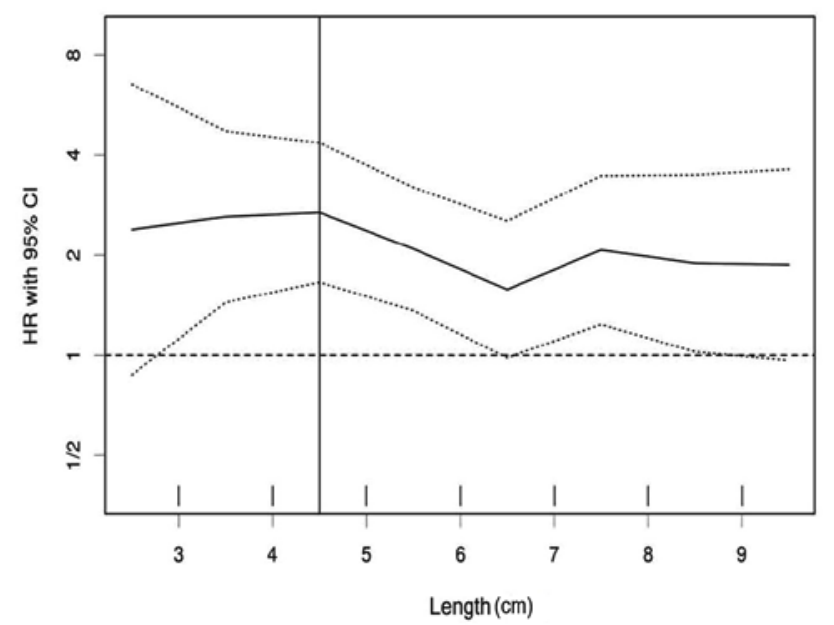

D

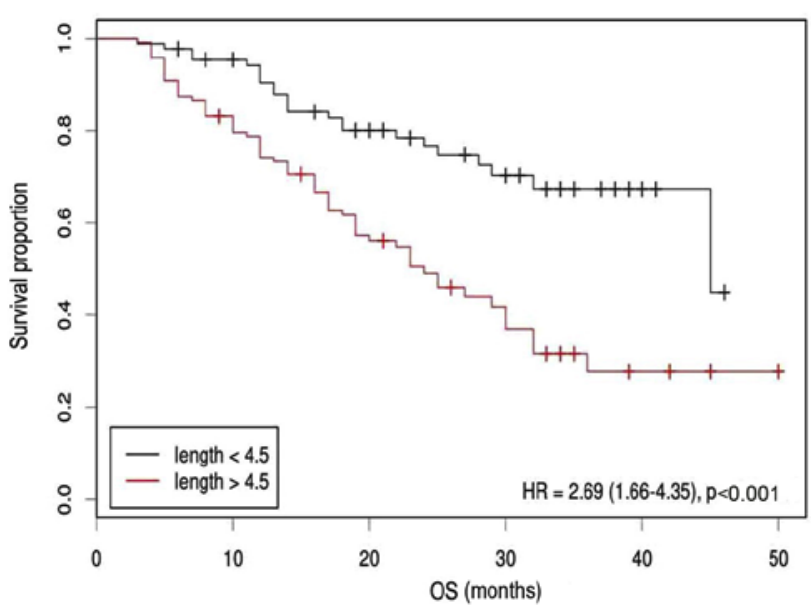

Figure 1. Cut-off optimization for tumor length associated with survival in the esophageal cancer data. (A) Tumor length in the 213 cases of esophageal cancer. The red vertical line designated the optimal cut-off derived from the survival-based model. (B) The HR, including 95\% CI, was plotted according to the cut-off. The vertical line designated the dichotomization showing the most significant association with OS. (C) Different OS according to tumor length (D) Difference in the mean OS according to tumor length. The red vertical line designated the dichotomization showing the most significant association with OS. CI, confidence interval; HR, hazard ratio; OS, overall survival.

(T) stage, regional lymph nodes $(\mathrm{N})$ stage, distant metastasis (M) stage and surgery. All tumors were staged according to the Tumor-Node-Metastasis (TNM) staging system of the American Joint Committee on Cancer (AJCC, 7th edition, 2009) (20). Furthermore, the cut-off value of tumor length affecting patient survival was determined using the Cut-off Finder application version 1.0 (http://molpath.charite. de/cutoff/) (21).

The primary endpoints of the present study were EC-SM and NEC-SM. EC-SM was defined as the mortality resulting from recurrent, metastatic or residual EC. Patients who exhibited EC progression at the last follow-up and patients who died from EC without a documented specific reason were included in the EC-specific group. NEC-SM was defined as the mortality resulting from specific causes other than EC, including death without a documented specific reason within 6 months of the last follow-up in the absence of EC relapse. The data from the surviving patients and from patients lost to follow-up were treated as censored data.
Patient follow-up. After IMRT treatment, all patients were followed at 3-month intervals for 2 years, at 6-month intervals for the next 3 years, and annually thereafter. The date of the last follow-up was July 2018. The last follow-up was mainly made by telephone calls. Recurrence was determined by clinical and radiological examination or histological confirmation. The main pattern of recurrence was recorded as the first site of detectable treatment failure during the follow-up period. The research endpoint corresponded to the overall survival (OS), which was calculated from the time of the diagnosis of EC until death from all causes or until the end of the follow-up period.

Statistical analysis. Clinicopathological characteristics were summarized using descriptive statistics. Patients were divided into two groups according to their age $(<60$ and $\geq 65$ years). EC-SM and NEC-SM were considered as two competing events, and the associations between pretreatment variables and the risk of EC-SM were evaluated using Fine and Gray's competing risk regression analysis (17). The 

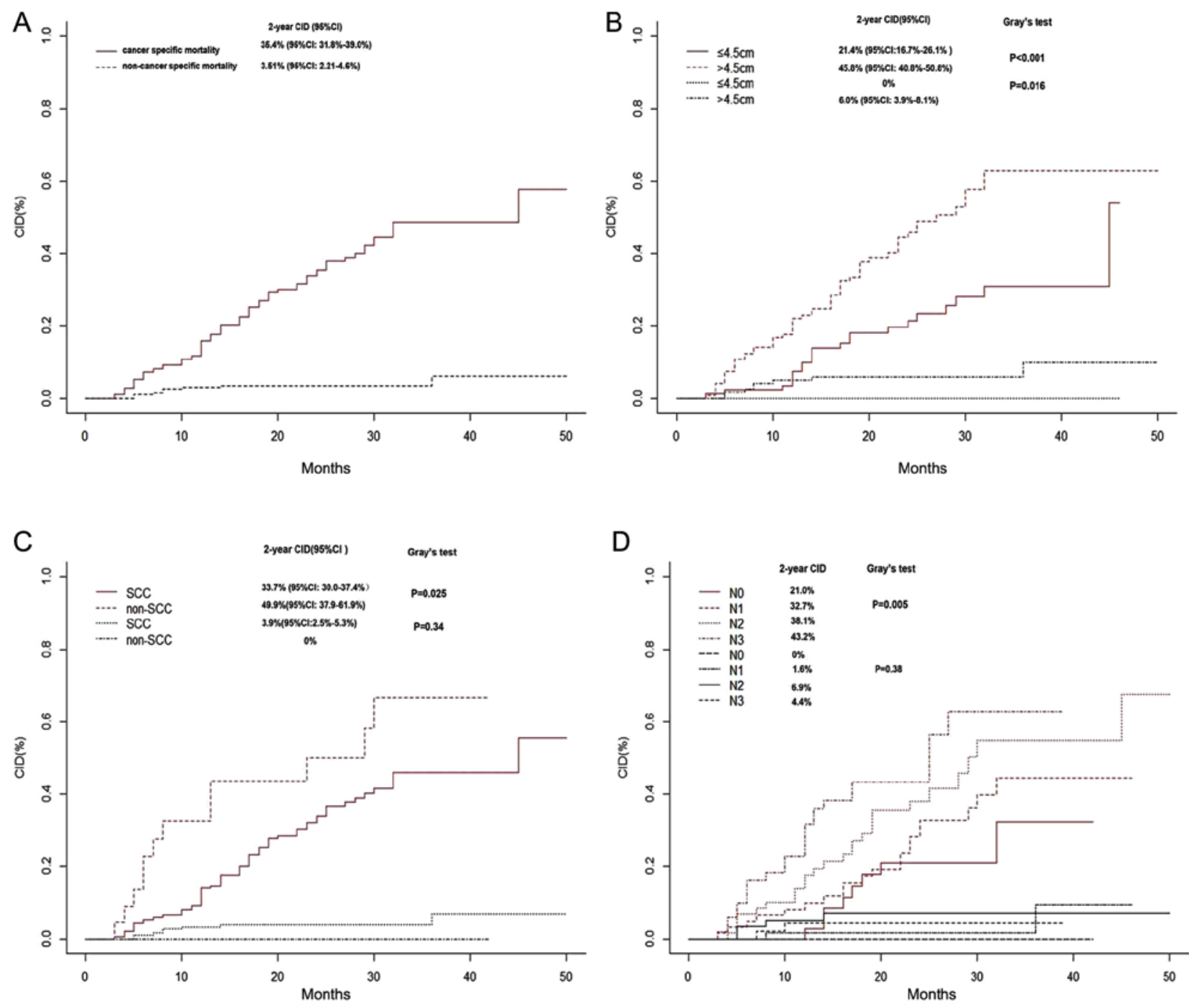

Figure 2. Cumulative incidence curves of deaths (A) in the entire cohort of patients with esophageal cancer and according to (B) tumor length, (C) histological type and (D) N stage. The 2-year cumulative incidences of each competing event were presented and the differences among the groups were estimated using Gray's test. The red line represented the cancer-specific mortality and the black line represented the competing mortality. CI, confidence interval; CID, cumulative incidence of death; SCC, squamous cell carcinoma.

cumulative incidence function (CIF) was used to determine the probability of each event, and the differences between the groups were estimated using Gray's test. Predictors with a $\mathrm{P}<0.05$ in the univariate analysis were used for multivariate analysis based on proportional sub-distribution hazard models (22). A nomogram predicting EC-specific survival (EC-SS) was constructed for patients with EC based on the identified independent risk factors. Nomograms were created using the nomogram function of the 'rms' package in $\mathrm{R}$ software (Mathsoft; version 3.6.1), the prediction performance was assessed using Harrell's concordance index (C-index) (23) and calibration curves were obtained by plotting the observed rates against the nomogram-predicted probabilities via a Bootstrap method with 1,000 resamples (17). The package 'partykit' in $\mathrm{R}$ was used to generate a decision tree (http://cran.salud.gob.sv/web/packages/partykit/vignettes/ctree.pdf). Overall survival was estimated using Kaplan-Meier method. Comparison between risk groups was performed by using log-rank test. Statistical analysis was performed using SPSS software (version 16.0;
SPSS Inc.). $\mathrm{P}<0.05$ was considered to indicate a statistically significant difference.

\section{Results}

Baseline characteristics. The clinicopathological characteristics of the 213 patients with EC included in the present study are summarized in Table I. The median age at diagnosis was 63 years (range, $41-87$ years). There were 183 men $(85.9 \%)$ and 30 women (14.1\%; male-to-female ratio, 6.1:1). The tumor histological types included squamous cell carcinoma $(89.7 \%)$ and adenocarcinoma $(8 \%)$ and other histological types $(2.3 \%)$. The tumor locations were thoracic $(61.5 \%)$, abdominal $(35.2 \%)$ and cervical (3.3\%). Concurrent chemoradiotherapy was administered to 172 patients $(80.8 \%)$, whereas 42 patients (19.2\%) were treated with IMRT alone due to advanced disease, comorbidities or patient requests. A total of 104 patients $(51.2 \%)$ received radical esophagectomy. The median length of the follow up was 19 months. In addition, 25 patients $(11.7 \%)$ presented with distant metastasis at initial diagnosis (Table I). 
Table II. Univariate and multivariate competing risk analyses of patient clinicopathological characteristics for the determination of esophageal cancer specific mortality.

\begin{tabular}{|c|c|c|c|c|c|c|}
\hline \multirow[b]{2}{*}{ Variable } & \multicolumn{3}{|c|}{ Univariate analysis } & \multicolumn{3}{|c|}{ Multivariate analysis } \\
\hline & SHR & $95 \%$ CI & P-value & SHR & $95 \% \mathrm{CI}$ & P-value \\
\hline \multicolumn{7}{|l|}{ Sex } \\
\hline Male & 1 & & & - & & \\
\hline Female & 0.68 & $0.34-1.37$ & 0.29 & - & - & - \\
\hline \multicolumn{7}{|l|}{ Age, years } \\
\hline$<60$ & 1 & & & - & & \\
\hline$\geq 60$ & 0.84 & $0.54-1.33$ & 0.47 & - & - & - \\
\hline \multicolumn{7}{|l|}{ Performance status } \\
\hline 0 & 1 & & & 1 & & \\
\hline $1-2$ & 1.66 & $1.04-2.65$ & 0.035 & 1.46 & $0.85-2.52$ & 0.17 \\
\hline \multicolumn{7}{|l|}{ Tumor location } \\
\hline Cervical & 1 & & & - & & \\
\hline Thoracic/abdominal & 0.91 & $0.58-1.42$ & 0.67 & - & - & - \\
\hline \multicolumn{7}{|l|}{ Histological types } \\
\hline $\mathrm{SCC}$ & 1 & & & 1 & & \\
\hline Non-SCC & 2.03 & $1.06-3.91$ & 0.034 & 2.71 & $1.48-4.93$ & 0.001 \\
\hline \multicolumn{7}{|l|}{ Grade } \\
\hline High & 1 & & & 1 & & \\
\hline Moderate/poor & 1.44 & $1.01-2.08$ & 0.046 & 1.14 & $0.75-1.74$ & 0.61 \\
\hline \multicolumn{7}{|l|}{ Surgery } \\
\hline No & 1 & & & 1 & & \\
\hline Yes & 0.62 & $0.40-0.96$ & 0.033 & 1.52 & $0.85-2.72$ & 0.15 \\
\hline \multicolumn{7}{|l|}{ Tumor length, $\mathrm{cm}$} \\
\hline$<4.5$ & 1 & & & 1 & & \\
\hline$\geq 4.5$ & 2.43 & $1.48-3.99$ & $<0.001$ & 1.80 & $1.03-3.16$ & 0.04 \\
\hline \multicolumn{7}{|l|}{ T stage } \\
\hline $\mathrm{T} 1$ & 1 & & & 1 & & \\
\hline $\mathrm{T} 2$ & 1.92 & $0.23-16.29$ & 0.55 & 1.42 & $0.16-12.49$ & 0.75 \\
\hline T3 & 3.39 & $0.45-25.49$ & 0.24 & 2.04 & $0.26-16.27$ & 0.50 \\
\hline $\mathrm{T} 4$ & 10.82 & $1.40-83.58$ & 0.022 & 5.37 & $0.59-49.29$ & 0.14 \\
\hline \multicolumn{7}{|l|}{$\mathrm{N}$ stage } \\
\hline No & 1 & & & 1 & & \\
\hline N1 & 1.51 & $0.71-3.24$ & 0.284 & 1.41 & $0.63-3.13$ & 0.41 \\
\hline $\mathrm{N} 2$ & 2.26 & $1.08-4.73$ & 0.030 & 1.71 & $0.76-3.82$ & 0.19 \\
\hline N3 & 3.31 & $1.54-7.09$ & 0.02 & 2.52 & $1.07-5.91$ & 0.034 \\
\hline \multicolumn{7}{|l|}{ Metastasis } \\
\hline No & 1 & & & 1 & & \\
\hline Yes & 2.12 & $1.08-4.13$ & 0.028 & 1.41 & $0.66-3.00$ & 0.37 \\
\hline \multicolumn{7}{|c|}{ Concurrent chemotherapy } \\
\hline Yes & 1 & & & - & - & - \\
\hline No & 1.20 & $0.68-2.12$ & 0.52 & - & - & - \\
\hline C-index (95\% CI) & - & - & - & - & $0.72(0.66-0.77)$ & - \\
\hline
\end{tabular}

CI, confidence interval; SHR, sub-distribution hazard ratio; SCC, squamous cell carcinoma; N, node; T, tumor.

A total of 23 patients $(10.8 \%)$ were lost to follow-up. In addition, 71 patients (33.3\%) died during follow-up, including
$65(91.5 \%)$ as a result of EC-SM and $6(8.5 \%)$ as a result of NEC-SM. Among the 6 NEC-SM patients, the most common 
Points

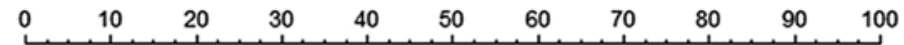

\section{Pathology}

Length

$\mathrm{N}$

Total points

\section{2-year cancer} specific survival
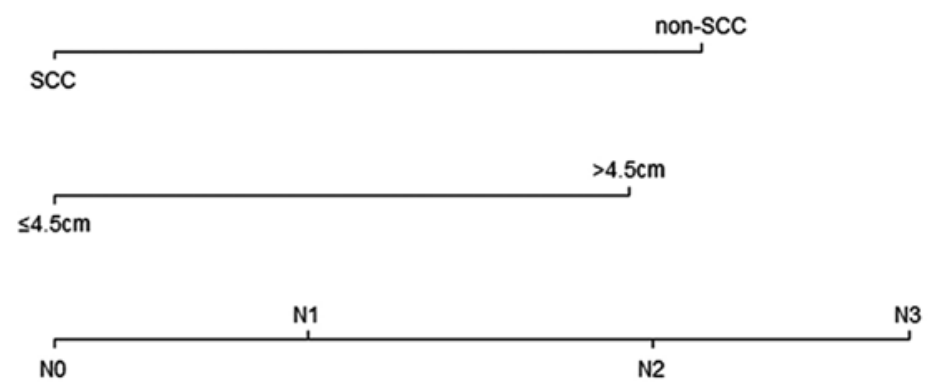

$0 \quad 50 \quad 100 \quad 150 \quad 200 \quad 300$

Figure 3. Competing risk nomograms for predicting the 2 -year probabilities of esophageal cancer-specific survival. Total point values were independently calculated for each cause of death and then applied to the corresponding probability scale at the bottom of each figure. N, lymph node stage; SCC, squamous cell carcinoma; $\mathrm{N}$, lymph node stage.

causes were treatment-associated complications (hemorrhage and pneumonia, 2 patients each; $66.7 \%$ account for NEC-SM) and cardiovascular and respiratory diseases (one patient each, respectively; $33.3 \%$ account for NEC-SM).

Survival outcomes of patients with EC following IMRT. The results of the cut-off point determination for primary tumor size indicated that $4.5 \mathrm{~cm}$ was the optimal point, which was supported by multiple methods of Cut-off Finder, including frequency (Fig. 1A), hazard ratio (Fig. 1B), difference in mean OS (Fig. 1C) and survival proportion (Fig. 1D). Furthermore, in patients with EC, the 2-year EC-SM and NEC-SM were 35.4 and $3.51 \%$, respectively (Fig. 2A). However, elevated 2-year EC-SM was observed in patients with tumor length $\geq 4.5 \mathrm{~cm}$ compared with patients with tumors length $<4.5 \mathrm{~cm}$ (45.8 vs. $21.4 \%$; $\mathrm{P}<0.001$; Fig. $2 \mathrm{~B}$ ), in patients with non-squamous cell carcinoma compared with patients with squamous cell carcinoma (49.9 vs. $33.7 \%$; $\mathrm{P}=0.025$; Fig. $2 \mathrm{C}$ ), and in patients with $\mathrm{N} 3$ stage in comparison with non-N3 stage (43.2\%; $\mathrm{P}=0.005$; Fig. 2D). The 2-year NEC-SM in patients with tumor length $\geq 4.5 \mathrm{~cm}$ was $6 \%$ (vs. $0 \%$ in $<4.5 \mathrm{~cm}$ group; $\mathrm{P}=0.016)$.

Riskfactors for EC-SM. The results from univariate competing risk regression analysis demonstrated that performance status [sub-distribution hazard ratio $(\mathrm{SHR})=1.66 ; 95 \%$ confidence interval $(\mathrm{CI}), 1.04-2.65 ; \mathrm{P}=0.035$, histological types $\mathrm{SHR}=2.03$; 95\% CI, 1.06-3.91; $\mathrm{P}=0.034]$, tumor grade $(\mathrm{SHR}=1.44 ; 95 \% \mathrm{CI}$, 1.01-2.08; $\mathrm{P}=0.046)$, surgery $(\mathrm{SHR}=0.62 ; 95 \% \mathrm{CI}, 0.40-0.96$; $\mathrm{P}=0.033)$, T4 stage $(\mathrm{SHR}=10.82 ; 95 \% \mathrm{CI}, 1.40-83.59 ; \mathrm{P}=0.022)$, $\mathrm{N} 2 / 3$ stage $(\mathrm{SHR}=2.26 ; 95 \% \mathrm{CI}, 1.08-4.73 ; \mathrm{P}=0.03$ and $\mathrm{SHR}=3.31 ; 95 \% \mathrm{CI}, 1.54-7.09 ; \mathrm{P}=0.02)$ and metastasis status $(\mathrm{SHR}=2.12 ; 95 \% \mathrm{CI}, 1.08-4.13 ; \mathrm{P}=0.028)$ were significantly associated with EC-SM (Table II). Multivariate analysis validated that tumor length $>4.5 \mathrm{~cm}(\mathrm{SHR}=1.80$; 95\% CI, 1.03-3.16;

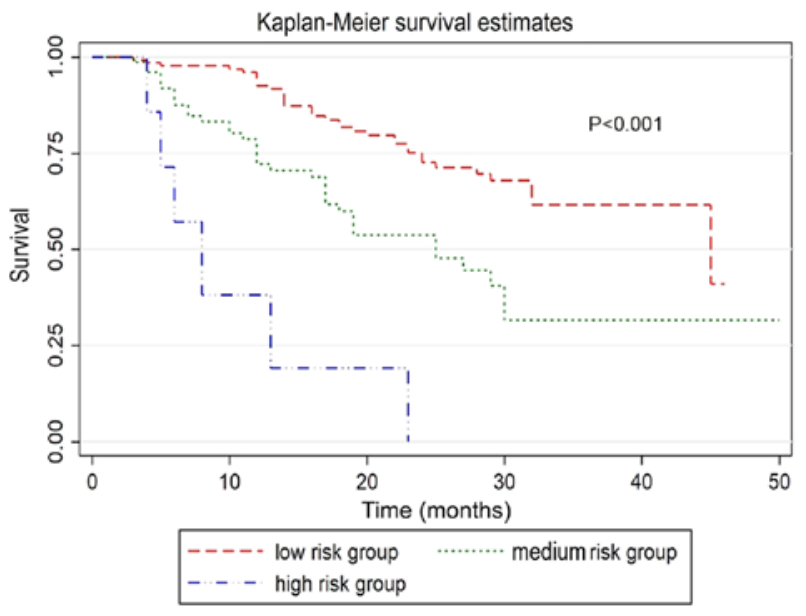

Figure 4. Esophageal cancer specific survival curves stratified according to the risk levels of the nomogram-predicted survival probabilities.

$\mathrm{P}=0.04)$, non-SCC $(\mathrm{SHR}=2.71 ; 95 \% \mathrm{CI}, 1.48-4.93 ; \mathrm{P}=0.001)$ and $\mathrm{N} 3$ stage $(\mathrm{SHR}=2.52 ; 95 \% \mathrm{CI}, 1.07-5.91 ; \mathrm{P}=0.034)$ were independent predictors for EC-SM (Table II).

Construction and validation of nomograms for EC-SS. All validated factors, including tumor length, histological type and $\mathrm{N}$ stage, were integrated into the competing nomograms in the EC-SS model (C-index $=0.72 ; 95 \%$ CI, 0.66-0.77; Fig. 3). The calibration plots for the probabilities of 2-year EC-SS demonstrated reasonable concordance between the nomogram-predicted survival rates and the actual survival rates in the training sets (Fig. S1). In addition, the nomograms exhibited improved discrimination power compared with the 7th edition of TNM staging system of AJCC for predicting EC-SS (area under the curve=0.707 vs. 0.634; Fig. S2). 


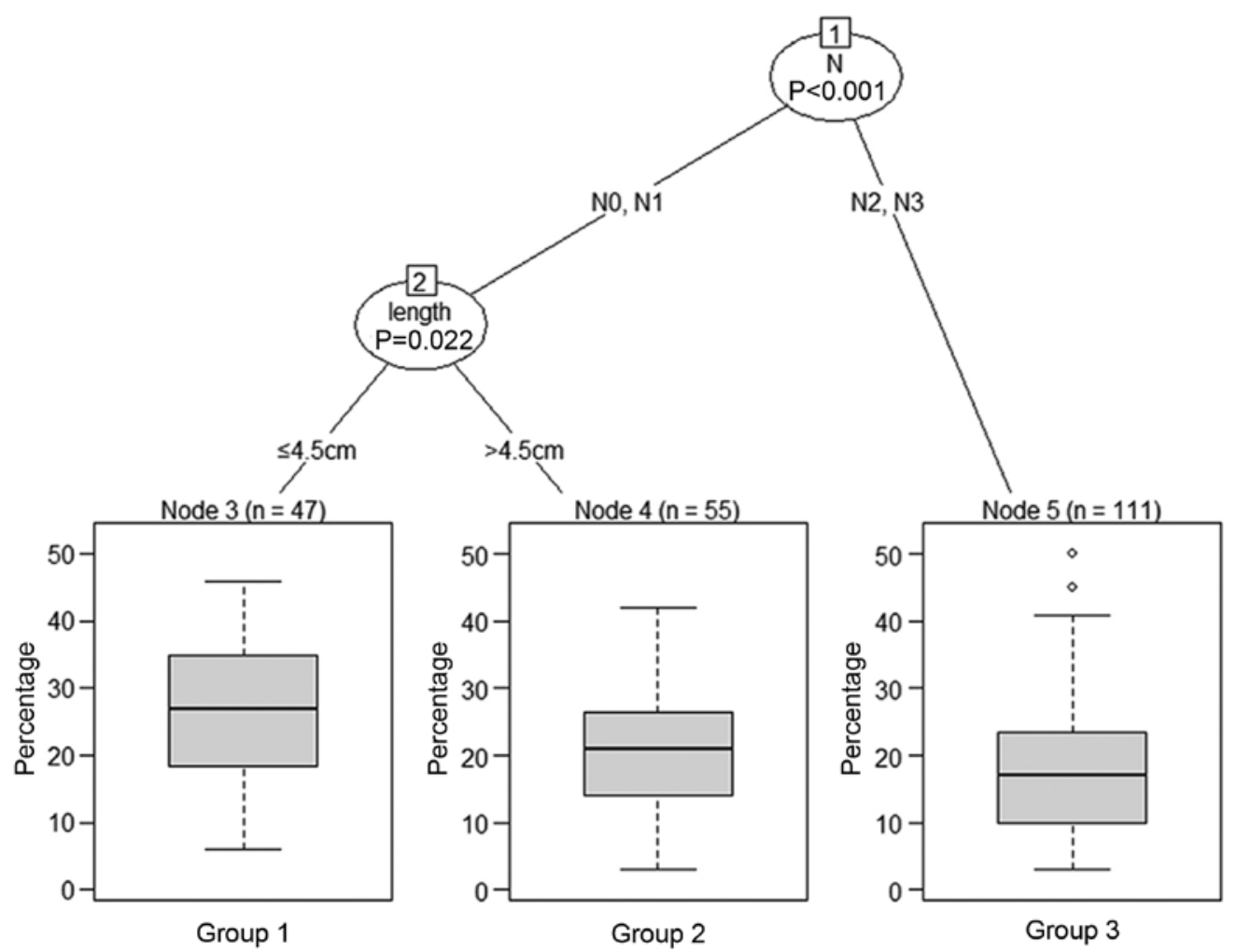

Figure 5. Decision tree for overall survival of patients with esophageal cancer. $\mathrm{N}$ stage was the initial node followed by tumor length. 1, initial node for survival. 2, second node for survival. N, lymph node stage.

Comparison of prognosis based on risk stratification. Each risk variable, including primary tumor size, $\mathrm{N}$ stage and histological type, were assigned values according to the nomogram for EC-SM (derived from the competing risk model). The total score was obtained by adding the individual scores of all significant parameters. Subsequently, three risk stratifications were defined as follows: Low risk (total score $\leq 100$ ), medium risk (>100 total score $\leq 200$ ) and high risk (total score $>200$ ). The results of the prognosis according to the risk stratification were as follows: 131 cases with low risk, 75 cases with medium risk and 7 cases with high risk. In addition, significant differences in OS $(\mathrm{P}<0.001)$ and EC-SM $(\mathrm{P}<0.001)$ were observed in the three risk groups (Fig. 4).

Classification tree for the factors associated with EC-SM. The classification tree analysis method was used to determine the factors associated with EC-SM following IMRT. The results of the pruned tree presented in Fig. 5 demonstrated that $\mathrm{N}$ stage was the initial node and that tumor length was also a determinant for EC-SM of these patients.

\section{Discussion}

In the present study, the 2-year EC-SM was 35.4\%, the median length of the follow up was 19 months, and $3.5 \%$ of patients died from causes other than EC. Since competing causes of mortality might have an impact on the prognosis of patients with EC, it is important to consider these competing risks when evaluating the prognosis in order to determine the best treatment option. To the best of our knowledge, the present study was the first to develop competing risk nomograms based on the proportional sub-distribution hazard method to predict EC-SM by using a cohort of patients with EC. The AJCC system, which depends on TNM staging, is the strongest clinical tool for the prognosis prediction of patients with EC. However, $\mathrm{T}$ classification represents the invasion depth of the tumor, which is different from the tumor length (24). Previous studies have reported existence of a link between tumor length and outcome in patients with EC (25-30). Similarly, the present study demonstrated that tumor length was an important prognostic factor for the EC-SM of patients who underwent IMRT. Furthermore, although most studies supported that tumor length is an important prognostic factor for EC, no consensus was achieved on the tumor size cut-off value, which ranges from 2 to $5 \mathrm{~cm}$ (31-33). The results from the present study demonstrated that elevated 2-year EC-SM was observed in patients with tumor length $\geq 4.5 \mathrm{~cm}(45.8 \%$ vs. $<4.5 \mathrm{~cm}$ group: $21.4 \%$; $\mathrm{P}<0.001)$. The $\mathrm{N}$ stage reflects regional lymph node metastasis, and previous studies reported that $\mathrm{N}$ stage is an important factor influencing the prognosis of patients with EC (23-25,34-36). Similarly, the present study demonstrated that the N3 stage was the strongest independent predictor for EC-SM, supporting the prognostic significance of the AJCC staging system in EC.

Esophageal SCC and esophageal non-SCC have distinct clinicopathological characteristics. The effects of the tumor histological type on the outcome of patients with EC were therefore investigated in the present study. The results demonstrated that the 2-year ES-SM in patients with non-SCC was significantly higher than in patients with SCC. This may be due to the fact that SCC tends to be more radiosensitive than non-SCC, particularly adenocarcinoma (37). 
The prognostic factors identified in the present study were integrated to develop nomograms that could predict the 2-year EC-SS of patients with EC. These nomograms displayed better discrimination power than the TNM staging system for predicting the EC-SS of patients with EC who received IMRT. Therefore, the nomograms developed in the present study may be used by radiation oncologists to precisely predict the individual patient's survival probability at specific time intervals.

The present study had some limitations. Firstly, only data of patients from one institution were included, which might limit the applicability of the nomogram. Secondly, the sample size was relatively small. Thirdly, the study was a retrospective study, and inherent bias could therefore not be excluded. Furthermore, different treatment strategies, including surgery and chemotherapy, may also affect the prognosis of patients with EC receiving IMRT. Finally, the results obtained in the present study were not externally validated. External validation is therefore required to determine whether the nomograms could be applied to other patient groups. Further evaluation in prospective multi-institute trials is therefore warranted.

In conclusion, the present study revealed that NEC-SM may represent a significant competing event for patients with EC and primary tumor length $\geq 4.5 \mathrm{~cm}$. Furthermore, to the best of our knowledge, the present study was the first to establish the first competing risk nomogram for EC that could be used to predict the probability of 2-year EC-SS. This nomogram may be considered as a convenient individualized predictive tool for determining the cancer-specific survival in patients with EC receiving IMRT.

\section{Acknowledgements}

Not applicable.

\section{Funding}

The present work was supported in part by Shanghai Integrated Traditional Chinese and Western Medicine Project (grant no ZHYY-ZXYJHZX-201913).

\section{Availability of data and materials}

The datasets used or analyzed during the present study are available from the corresponding author on reasonable request.

\section{Authors' contributions}

SZ and WQ designed the study. WQ, SZ and JC analyzed and interpreted the patient data. SZ was a major contributor in writing the manuscript. All authors read and approved the final manuscript.

\section{Ethics approval and consent to participate}

The study was approved by the Institutional Review Board at Shanghai Rui Jin Hospital Affiliated to Shanghai Jiao Tong University School of Medicine. Written informed consent was obtained from all individual participants included in the study.

\section{Patient consent for publication}

Not applicable.

\section{Competing interests}

The authors declare that they have no competing interests.

\section{References}

1. Bray F, Ferlay J, Soerjomataram I, Siegel RL, Torre LA and Jemal A: Global cancer statistics 2018: GLOBOCAN estimates of incidence and mortality worldwide for 36 cancers in 185 countries. CA Cancer J Clin 68: 394-424, 2018.

2. Domper Arnal MJ, Ferrandez Arenas A and Lanas Arbeloa A: Esophageal cancer: Risk factors, screening and endoscopic treatment in Western and Eastern countries. World J Gastroenterol 21: 7933-7943, 2015.

3. Chen W, Zheng R, Baade PD, Zhang S, Zeng H, Bray F, Jemal A, Yu XQ and He J: Cancer statistics in China, 2015. CA Cancer J Clin 66: 115-132, 2016.

4. Zheng R, Zeng H, Zhang S and Chen W: Estimates of cancer incidence and mortality in China, 2013. Chin J Cancer 36: 66, 2017.

5. Sohda M and Kuwano H: Current status and future prospects for esophageal cancer treatment. Ann Thorac Cardiovasc Surg 23: 1-11, 2017.

6. Berry MF: Esophageal cancer: Staging system and guidelines for staging and treatment. J Thorac Dis 6: S289-S297, 2014.

7. $\mathrm{Xi} \mathrm{M}$ and Lin SH: Recent advances in intensity modulated radiotherapy and proton therapy for esophageal cancer. Expert Rev Anticancer Ther 17: 635-646, 2017.

8. Fenkell L, Kaminsky I, Breen S, Huang S, Van Prooijen M and Ringash J: Dosimetric comparison of IMRT vs. 3D conformal radiotherapy in the treatment of cancer of the cervical esophagus. Radiother Oncol 89: 287-291, 2008.

9. Bollschweiler E, Plum P, Monig SP and Holscher AH: Current and future treatment options for esophageal cancer in the elderly. Expert Opin Pharmacother 18: 1001-1010, 2017.

10. Berry SD, Ngo L, Samelson EJ and Kiel DP: Competing risk of death: An important consideration in studies of older adults. J Am Geriatr Soc 58: 783-787, 2010.

11. Zhang Z, Geskus RB, Kattan MW, Zhang H and Liu T: Nomogram for survival analysis in the presence of competing risks. Ann Transl Med 5: 403, 2017.

12. Carmona R, Zakeri K, Green G, Hwang L, Gulaya S, Xu B, Verma R, Williamson CW, Triplett DP, Rose BS, et al: Improved method to stratify elderly patients with cancer at risk for competing events. J Clin Oncol 34: 1270-1277, 2016.

13. Simpson MC, Massa ST, Boakye EA, Antisdel JL, Stamatakis KA, Varvares MA and Osazuwa-Peters N: primary cancer vs. competing causes of death in survivors of head and neck cancer. JAMA Oncol 4: 257-259, 2018.

14. Eguchi T, Bains S, Lee MC, Tan KS, Hristov B, Buitrago DH, Bains MS, Downey RJ, Huang J, Isbell JM, et al: Impact of increasing age on cause-specific mortality and morbidity in patients with stage I non-small-cell lung cancer: A competing risks analysis. J Clin Oncol 35: 281-290, 2017.

15. Grobman WA and Stamilio DM: Methods of clinical prediction. Am J Obstet Gynecol 194: 888-894, 2006.

16. Kutikov A, Egleston BL, Wong YN and Uzzo RG: Evaluating overall survival and competing risks of death in patients with localized renal cell carcinoma using a comprehensive nomogram. J Clin Oncol 28: 311-317, 2010.

17. Huang XD, Zhou GQ, Lv JW, Zhou HQ, Zhong CW, Wu CF, Zheng ZQ, He XJ, Peng L, Ma J and Sun Y: Competing risk nomograms for nasopharyngeal carcinoma in the intensity-modulated radiotherapy era: A big-data, intelligence platform-based analysis. Radiother Oncol 129: 389-395, 2018.

18. Luo C, Zhong X, Deng L, Xie Y, Hu K and Zheng H: Nomogram predicting locoregional recurrence to assist decision-making of postmastectomy radiotherapy in patients with T1-2N1 breast cancer. Int J Radiat Oncol Biol Phys 103: 905-912, 2019. 
19. Yang L, Shen W and Sakamoto N: Population-based study evaluating and predicting the probability of death resulting from thyroid cancer and other causes among patients with thyroid cancer. J Clin Oncol 31: 468-474, 2013.

20. Edge SB and Compton CC: The American Joint Committee on Cancer: The 7th edition of the AJCC cancer staging manual and the future of TNM. Ann Surg Oncol 17: 1471-1474, 2010.

21. Li X, Wan J, Wu Z, Tu J, Hu Y, Wu S and Lou L: Fatal adverse events with molecular targeted agents in the treatment of advanced hepatocellular carcinoma: A meta-analysis of randomized controlled trials. Drug Des Devel Ther 12: 3043-3049, 2018.

22. Sfumato P, Filleron T, Giorgi R, Cook RJ and Boher JM: Goftte: A R package for assessing goodness-of-fit in proportional (sub) distributions hazards regression models. Comput Methods Programs Biomed 177: 269-275, 2019.

23. Chen Y, Jia Z, Mercola D and Xie X: A gradient boosting algorithm for survival analysis via direct optimization of concordance index. Comput Math Methods Med 2013: 873595, 2013.

24. Hong SM, Cho H, Moskaluk CA and Yu E: Measurement of the invasion depth of extrahepatic bile duct carcinoma: An alternative method overcoming the current $\mathrm{T}$ classification problems of the AJCC staging system. Am J Surg Pathol 31: 199-206, 2007.

25. Zhang X, Wang Y, Qu P, Liu-Helmersson J, Zhao L, Zhang L and Sang S: Prognostic value of tumor length for cause-specific death in resectable esophageal cancer. Ann Thorac Surg 106: 1038-1046, 2018

26. Zhang X, Wang Y, Jiang Y, Wang Z, Zhao L, Xue X, Sang S and Zhang L: The prognostic impact of tumor length in esophageal cancer: Protocol for a systematic review and meta-analysis. Medicine (Baltimore) 97: e12902, 2018.

27. Wang BY, Liu CY, Lin CH, Hsu PK, Hsu WH, Wu YC and Cheng CY: Endoscopic tumor length is an independent prognostic factor in esophageal squamous cell carcinoma. Ann Surg Oncol 19: 2149-2158, 2012.

28. Gaur P, Sepesi B, Hofstetter WL, Correa AM, Bhutani MS, Watson TJ, Swisher SG, M. D. Anderson Esophageal Cancer Group and University of Rochester School of Medicine and Dentistry Foregut Group: Endoscopic esophageal tumor length: A prognostic factor for patients with esophageal cancer. Cancer 117: 63-69, 2011.

29. Koyanagi K, Kato F, Kanamori J, Daiko H, Ozawa S and Tachimori Y: Clinical significance of esophageal invasion length for the prediction of mediastinal lymph node metastasis in Siewert type II adenocarcinoma: A retrospective single-institution study. Ann Gastroenterol Surg 2: 187-196, 2018.
30. Arigami T, Uchikado Y, Omoto I, Sasaki K, Kita Y, Owaki T, Yanagita S, Mori S, Kurahara H, Okumura H, et al: Primary tumor score based on tumor depth and length predicts prognosis in esophageal squamous cell carcinoma. Anticancer Res 38: 5447-5452, 2018.

31. Zhang X, Wang Y, Li C, Helmersson J, Jiang Y, Ma G, Wang G, Dong W, Sang S and Du J: The prognostic value of tumor length to resectable esophageal squamous cell carcinoma: A retrospective study. Peer J 5: e2943, 2017.

32. Vadhwana B, Zosimas D, Lykoudis PM, Phen HM, Martinou M and Khoo D: Tumour length as an independent prognostic factor in resectable oesophageal carcinoma. Ann R Coll Surg Engl 22: $1-6,2019$.

33. $\mathrm{Xu} \mathrm{H}, \mathrm{Wu} \mathrm{S}$, Luo $\mathrm{H}$, Chen $\mathrm{C}$, Lin $\mathrm{L}$, Huang $\mathrm{H}$ and Xue R: Prognostic value of tumor length and diameter for esophageal squamous cell cancer patients treated with definitive (chemo) radiotherapy: Potential indicators for nonsurgical $\mathrm{T}$ staging. Cancer Med 8: 6326-6334, 2019.

34. Yin $\mathrm{H}$, Li D, Zhu C, Wang $\mathrm{M}$ and Wei N: Factors relevant to the prognosis of patients with esophageal cancer who received intensity-modulated radiotherapy. Thorac Cancer 9: 1215-1219, 2018.

35. Shiozaki H, Sudo K, Xiao L, Wadhwa R, Elimova E, Hofstetter WL, Skinner HD, Lee JH, Weston B, Bhutani MS, et al: Distribution and timing of distant metastasis after local therapy in a large cohort of patients with esophageal and esophagogastric junction cancer. Oncology 86: 336-339, 2014.

36. Sakanaka K, Ishida Y, Itasaka S, Ezoe Y, Aoyama I, Miyamoto S, Horimatsu T, Muto $\mathrm{M}$ and Hiraoka M: Identification of a predictive factor for distant metastasis in esophageal squamous cell carcinoma after definitive chemoradiotherapy. Int J Clin Oncol 21: 899-908, 2016.

37. Chen GZ, Zhu HC, Dai WS, Zeng XN, Luo JH and Sun XC: The mechanisms of radioresistance in esophageal squamous cell carcinoma and current strategies in radiosensitivity. J Thorac Dis 9: 849-859, 2017.

This work is licensed under a Creative Commons Attribution-NonCommercial-NoDerivatives 4.0 International (CC BY-NC-ND 4.0) License. 